\title{
Data-Driven Medicine: A Meaningful Use of Patient Data to Improve Process Adherence and Patient Outcome of Mechanically Ventilated Subjects
}

In this issue of RESPIRATORY CARE, Walsh et $\mathrm{al}^{1}$ report on the implementation of a data visualization tool that translates the overwhelming amount of patient data originating from various sources in the ICU into meaningful clinical information. By their intervention, the authors primarily intend to improve adherence to evidence-based guidelines during invasive mechanical ventilation in their pediatric ICU population. In this editorial, we discuss the clinical and technical challenges and potential benefits of the authors' intervention.

Evidence-based guidelines are only meaningful if their implementation leads to the translation of medical evidence in a feasible clinical process. Unfortunately, there is often a discrepancy between evidence-based recommendations and clinical practice. ${ }^{2}$ Why does this happen in the real world? Various reasons have been put forward, but one important reason is the inability to apply the evidence in clinical practice. ${ }^{3}$ Furthermore, the lack of rigorous process adherence remains problematic and might contribute to worse outcomes for the patient. ${ }^{4}$ This is in sharp contrast with our increasing capabilities to monitor the clinical process in detail..$^{5}$ Especially in the ICU, we capture on a continuous basis a considerable amount of data from various devices (eg, monitoring devices, mechanical ventilators, dialysis machines). These data are directly communicated to our electronic medical record. Our ability to document and monitor in the ICU the clinical process in such detail has never been more evident than today.

Despite the overwhelming amount of data, clinicians often fail to translate this stream of data into meaningful information that is relevant for the clinical context of the individual patient. Also, despite the continuous character of the data, clinicians often keep using the electronic medical record as an old-fashioned paper record relying on (randomly) one or 2 hourly point prevalence measure-

The authors have disclosed no conflicts of interest.

Correspondence: Walter Verbrugghe MD, Department of Critical Care Medicine, Antwerp University Hospital, Edegem, Belgium. E-mail: Walter.Verbrugghe@uza.be.

DOI: $10.4187 /$ respcare. 05457 ments, neglecting the full continuum of the data. ${ }^{6}$ Appreciating that significant physiological alterations might occur during short time periods, there is a need to take the whole continuum of the available data into account in our clinical decision making. ${ }^{7,8}$

\section{See the Original Study on Page 268}

The study by Walsh et $\mathrm{al}^{1}$ published in the current issue illustrates a considerable effort to tackle this problem in the field of mechanical ventilation. By using evidencebased treatment goals for pediatric patients receiving invasive mechanical ventilation as a starting point, the authors showed how process adherence during mechanical ventilation can be improved. An essential element in their strategy was to integrate the data from connected mechanical ventilators and other medical equipment with the clinical information already available in the electronic medical record system. The data were processed by a software tool and visualized in a highly informative format to the clinician. This allowed the clinician to monitor and track the process of mechanical ventilation in his or her patients.

The authors were innovative by capturing the data on a continuous basis, by integrating multiple data sources, and by transforming this overwhelming information load into useful clinical information. Remarkably, a beneficial effect was shown on different outcomes, such as acceptable ventilation, acceptable oxygenation, barotrauma, and volutrauma-free ventilation time. The largest improvement was achieved in the volutrauma-free category. This is of significance because volutrauma plays a crucial role in the development of ventilator-induced lung injury. ${ }^{9}$ The continuous character of the data visualization might be of clinical importance because even short periods of inadequate mechanical ventilation might affect patient outcome. ${ }^{10}$ Finally, the authors attempted to integrate all of the data into a single parameter, the mechanical ventilation score. This score showed improvement during the study and tended to be higher in survivors.

The study presented in this issue might be criticized for complexity, since many simultaneous interventions took place. It is also clear that the mechanical ventilation score 
will need additional prospective validation. Also, the study was not powered adequately to detect a difference in clinical outcome.

Additionally, one particular point that needs specific attention is the management of artifacts. ${ }^{11}$ A significant proportion of an automatically measured vital parameter (eg, blood pressure measurement or transcutaneous oxygen saturation) might be artifactual. ${ }^{12}$ It is crucial that these artifacts be well recognized and dealt with by the computer to provide the clinician with reliable information. Another point is that the authors had to develop a separate software tool. This illustrates that contemporary (enterprise) electronic medical records or (stand-alone) patient data management systems still do not provide all of the tools to accomplish this task. Ideally, this kind of functionality should be built into the electronic medical record or patient data management system.

Nevertheless, the authors demonstrated well that process adherence increased as a result of their interventions. This is still no guarantee of better patient outcome, but at least it allows the clinician to make an informed, and hopefully better, treatment decision. The authors acknowledge that the hospital of the future is a data-driven hospital. The authors have shown that this is also applicable in the pediatric ICU, at least in the field of mechanical ventilation, but also in many other fields of intensive care. The next major future challenge is to optimize the use of this growing quantity of medical data by translating these data into meaningful clinical information that enables the clinician to improve clinical decision making during ventilation.

\section{REFERENCES}

1. Walsh BK, Smallwood C, Rettig J, Kacmarek RM, Thompson J, Arnold JA. Daily goals formulation and enhanced visualization of mechanical ventilation variance improves mechanical ventilation score. Respir Care 2017;62(3):268-278.

2. Buscaglia J, Nagula S, Yuan J, Bucobo JC, Kumar A, Forsmark CE, Draganov PV. The practice of evidence-based medicine (EBM) in gastroenterology: discrepancies between EBM familiarity and EBM competency. Therap Adv Gastroenterol 2011;4(5):283-294.

3. Haynes B, Haines A. Getting research findings into practice: Barriers and bridges to evidence based clinical practice. BMJ 1998;317(7153): 273-276.

4. Gao F, Melody T, Daniels DF, Giles S, Fox S. The impact of compliance with 6-hour and 24-hour sepsis bundles on hospital mortality in patients with severe sepsis: a prospective observational study. Crit Care 2005;9(6):R764-R770.

5. Herasevich V, Litell J, Pickering B. Electronic medical records and mHealth anytime, anywhere. Biomed Instrum Technol 2012. doi: 10.2345/0899-8205-46.s2.45.

6. Maslove DM, Dubin JA, Shrivats A, Lee J. Errors, omissions, and outliers in hourly vital signs measurements in intensive care. Crit Care Med 2016;44(11):e1021-e1030.

7. Norris PR, Dawant BM. Closing the loop in ICU decision support: physiologic event detection, alerts, and documentation. Proc AMIA Symp 2001;498-502.

8. Evans RS, Kuttler KG, Simpson KJ, Howe S, Crossno PF, Johnson $\mathrm{KV}$, et al. Automated detection of physiologic deterioration in hospitalized patients. J Am Med Inform Assoc 2015;22(2):350-360.

9. Beitler JR, Malhotra A, Thompson BT. Ventilator-induced lung injury. Clin Chest Med 2016;37(4):633-646.

10. Ladha K, Vidal Melo MF, McLean DJ, Wanderer JP, Grabitz SD, Kurth T, Eikermann M. Intraoperative protective mechanical ventilation and risk of postoperative respiratory complications: hospital based registry study. BMJ 2015;351:h3646.

11. Cao H, Norris P, Ozdas A, Jenkins J, Morris JA. A simple nonphysiological artifact filter for invasive arterial blood pressure monitoring: a study of 1852 trauma ICU patients. Conf Proc IEEE Eng Med Biol Soc 2006;1:1417-1420.

12. Clifford GD, Long WJ, Moody GB, Szolovits P. Robust parameter extraction for decision support using multimodal intensive care data. Phil Trans R Soc A 2009;367(1887):411-429. 